Journal of Zhejiang University-SCIENCE C (Computers \& Electronics)

ISSN 1869-1951 (Print); ISSN 1869-196X (Online)

www.zju.edu.cn/jzus; www.springerlink.com

E-mail: jzus@zju.edu.cn

\title{
Editorial:
}

\section{Recent advances of the Semantic Web}

\section{Zhao-hui WU}

Guest Editor-in-Chieffor this special issue

Zhejiang University, Hangzhou 310027, China

E-mail: wzh@zju.edu.cn

doi: $10.1631 /$ jzus.C1101000

The World Wide Web (WWW) has become an indispensable media in our daily life. It is frequently used in our day-to-day operations to procure a solution for a difficult problem, communicate and socialize with others, reserve hotels and book tickets to arrange our trips, seek business opportunities, entertain ourselves, and so forth. Without the Web, our life may not have been what it is today. However, a number of questions remain: Has the Web reached its full potential? Can it change our life more than what we have seen? What will the Web look like 20 years from now? One key question out of all is: Could the Web be smarter and more intelligent than before, considering the overwhelming Web content generated at such an exponential rate?

The Semantic Web is widely recognized as the most important enabling technology that can help realize a smarter Web and enable Web intelligence. The term Semantic Web was coined by Web inventor Tim Berners-Lee, and is an extension of the current Web. It is described as a giant global Web of data that is directly consumable and understandable to machines. In contrast to a hypertext Web that indicates texts linked to texts in other places by hyperlinks, the Semantic Web projects a hyperdata Web that indicates data objects linked with other data objects across the Web through formal semantics and ontologies.

(C) Zhejiang University and Springer-Verlag Berlin Heidelberg 2012
In December 2011, researchers from all over the world gathered together in Hangzhou, China, for the 1st Joint International Semantic Technology Conference (JIST2011). JIST2011 is a joint event for regional Semantic Web related conferences. This year's JIST brought together two regional conferences: ASWC2011 (Asian Semantic Web Conference 2011) and CSWC2011 (5th Chinese Semantic Web Conference).

As a follow up to this successful event, this special issue aims to promote the discussion on current trends of the Semantic Web. Our goal is not only to select the best papers from the conference, but also to present cutting-edge perspectives and visions to highlight future developments. Taking this into account, we have organized this special issue with many new features. Structurally, it has the following four components: Perspectives, Personal Views, Research Articles, and Application Reports.

For the perspective part, we invited Professor Ian Horrocks from Oxford University and Professor Riichiro Mizoguchi from Osaka University to present their own perspectives, with a particular focus on the scalability issue of the Semantic Web. Professor Ian Horrocks reviews the evolution of semantic technologies to date, and then examines the scalability challenges that arise from deployment in large-scale applications. Professor Riichiro Mizoguchi emphasizes the exact meaning of 'scalability' of the Semantic Web data, from the perspective of the data on the WWW scale and Linked Data scale.

For a personal view, Mark Greaves from Vulcan presents his view on two properties of the Semantic Web: how existing Internet social ('crowd') phenomena can apply to data on the Semantic Web, and how we can use these social Web techniques to 
improve the dynamic scalability of the Semantic Web. Dr. Jeff Z. Pan from the University of Aberdeen presents his personal view on an important problem in many Semantic Web applications, i.e., local closed world reasoning in ontologies. He also proposes several topics related to this research area and discusses possible technical directions. Professor Zhao-hui Wu presents his personal view on the technical evolution of the Semantic Grid into Knowledge Service Cloud. He states that Knowledge Service Cloud is the future e-Science infrastructure that emphasizes supporting knowledge creation activities with intelligent services, anytime, anywhere, and via any device.

The research articles part is composed of four selected articles. The first article proposes a MapReduce-based approach to tackle scalability challenge for matching large ontologies, for instance, long run time or strong hypotheses on the running environment. The second article proposes an interesting approach for building a large-scale Chinese structured knowledge base from Chinese wiki resources, including Hudong and Baidu Baike. The third article is a study on improving SPARQL query performance with semantic caching approaches, i.e., SPARQL algebraic expression tree (AET) based caching and entity caching. The fourth article presents a multi-agent framework for mining hypothetical semantic relations from the Linked Data. These agents collaborate in relation mining by publishing and exchanging inter- dependent knowledge elements, e.g., hypotheses, evidence, and proofs, giving rise to an evidentiary network that connects and ranks diverse knowledge elements.

To encourage and promote the adoption of Semantic Web technologies, the last part of this special issue is devoted to reports on typical applications. The first one from Oracle specifically reports on enterprise applications of semantic technologies for business process management. The second one introduces a featured application in traditional Chinese medicine
(TCM), and describes an in-use national semantic infrastructure developed for TCM communities.

As another novel feature of this special issue, we append a list of related articles recommended by the authors at the end of the papers, where space is available, so that readers can easily follow on specific topics based on these suggested readings.

Lastly, we would like to express our appreciation to our distinguished reviewers, whose expertise and professionalism has contributed significantly to the quality of this special issue. We would also like to thank Helen (Y.H.) Zhang, managing editor of Journals of Zhejiang University-SCIENCE $(A / B / C)$, and Zi-yang Zhai, editor of this special issue, for their helpful guidance and constructive feedback in the entire process of organizing and publishing this special issue.

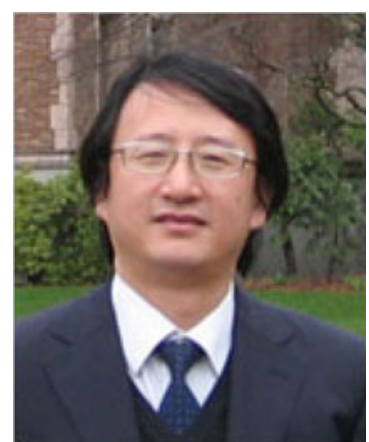

Zhao-hui Wu, Associate Editor-in-Chief of $J Z U S-C$, is a professor of the School of Computer Science and Technology, Zhejiang University, China. In the 1990s, Professor Wu invented the first KB-system tool, ZIPE, and the first coupling knowledge representing model, Couplingua, which embodies Rule, Frame, Semantic Network, and Nerve Cell Network. In the 2000s, he led the development of a Semantic Grid platform called DartGrid. He also took a lead in developing TCM-Grid, the world-largest data sharing infrastructure for traditional Chinese medicine. His current research focus includes big-data computing, Semantic Web, cloud computing, and their applications in healthcare and traditional Chinese medicine. 